\title{
Quantitative Anatomical Approaches to Examining Plasticity in Neural Circuits
}

\author{
Deborah M. Hegarty ${ }^{1}$, Angela E. Gonzalez ${ }^{2}$, John H. Harkness ${ }^{2}$, Barbara A. Sorg ${ }^{2}$, Sue A. Aicher ${ }^{1 *}$ \\ 1. Oregon Health \& Science University, Physiology \& Pharmacology, Portland, OR, USA. \\ 2. Washington State University, Dept. of Integrative Physiology and Neuroscience, Vancouver, WA, \\ USA. \\ * Corresponding author: aichers@ohsu.edu
}

Drugs of abuse can cause dynamic changes in brain circuits that may sustain learning that helps maintain habitual drug use. Our studies use a multi-disciplinary approach to quantitatively examine specific cells in the brain that are involved in sustaining addictive behaviors. By examining changes in neuronal content of neurotransmitters, changes in neuronal activity, and changes in synaptic input, we hope to gain a deeper understanding of the effects of drugs of abuse on neural circuits.

Our studies focus on cells in the medial prefrontal cortex of the rat brain that are vital for drug-induced habit formation. We specifically examined inhibitory neurons in the cortex that contain parvalbumin (PV), a calcium-binding protein. Because PV cells send projections to numerous neurons in the cortex, changes in the activity of these inhibitory PV cells can alter the activity of the circuit that modulates learning, including learning associated with drug cues.

In addition to containing PV, many of these cells are surrounded by perineuronal nets (PNNs). PNNs are extracellular matrix materials that are important in regulating critical periods of neural circuit development, but also appear to show plasticity in response to drug administration. Our hypothesis is that drugs of abuse alter PNNs and allow changes in synaptic input to the underlying PV neurons.

Our recent study [1] showed that cocaine administration can acutely alter the density of the PNNs around PV cells, as well as the number and types of inputs the PV cells receive. We quantified excitatory inputs using immunocytochemical detection of glutamate vesicular transporters (VGluT) as well as inhibitory inputs using immunocytochemical detection of glutamic acid decarboxylase (GAD). Each antibody was detected with a secondary antibody with a distinct fluorophore. PNNs were detected with binding of Wisteria floribunda agglutinin (WFA) and visualised with streptavidin conjugated to a fluorophore. High magnification images were collected by confocal microscopy (Fig. 1A) and then optical sections through the middle of each PV cell body were analysed using Imaris software. The PV cell body was segmented with the Imaris Surfaces segmentation tool, creating a 3-dimensional rendering of each cell through its middle (Fig. 1B, C). A $1.5 \mu \mathrm{m}$ area immediately surrounding the PV cell surface and following its unique contours was created to limit the processing time required for GAD and VGluT analysis and increase workflow. The Mask Channel Function was then utilized to isolate GAD- and VGluT-labeled inputs within the $1.5 \mu \mathrm{m}$ perimeter. Distinct appositions onto the PV cell surface from inhibitory (GAD) or excitatory (VGluT) axonal inputs were segmented using the Imaris Spots segmentation tool and refined with an Imaris Xtension function to isolate those spots close to the Imarisgenerated PV cell surface (Fig. 1C). This analytic approach allows quantification of anatomical inputs to each cell body as well as calculation of ratios of inputs. Together with examination of behavioral changes and altered electrophysiological responses of PV cells, we are gaining a greater understanding of the changes in neuronal circuitry caused by drugs of abuse. Recent studies [1] show that 1 or 5 days of cocaine exposure can cause signification changes in the anatomy and activity of this circuit. 
Emerging findings suggested that some of this anatomical plasticity may be due to learning, such that even exposure to the environment where drugs were administered may alter this brain circuit [2].

\section{References:}

[1] Slaker ML, et al., eNeuro 5(5) (2018) doi: 10.1523/ENEURO.0221-18.2018.

[2] The authors acknowledge funding from the National Institute of Drug Abuse (R01 DA040965) and the National Institutes of Neurological Disorders and Stroke (P30 NS016800).
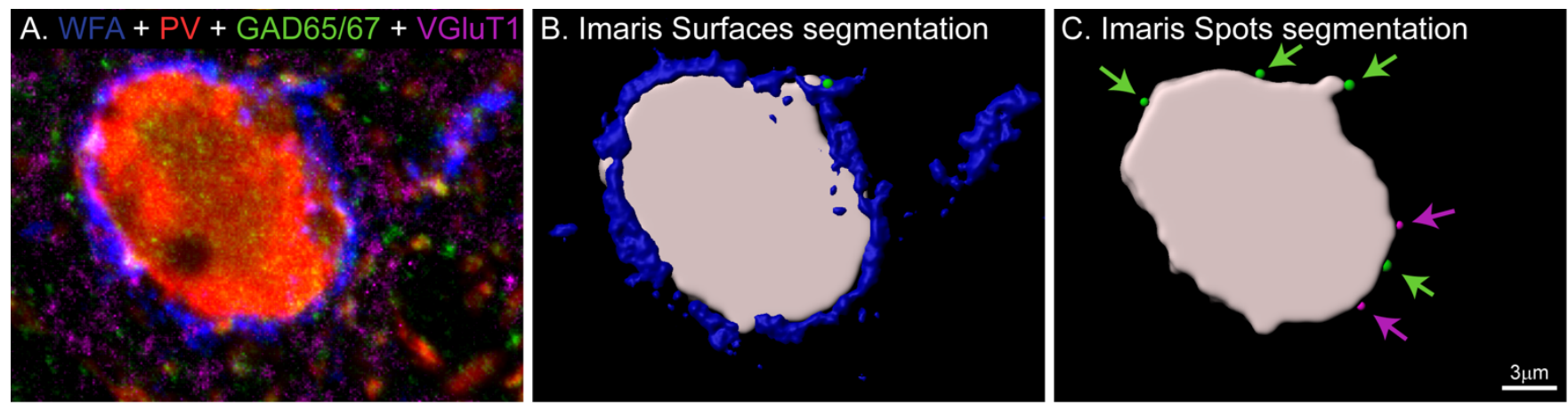

Figure 1. A. Neurons in the medial prefrontal cortex of the rat contain parvalbumin (red) and are surrounded by perineuronal nets (blue). These cells receive input from axons of other neurons that contained either excitatory neurotransmitters (VGluT, magenta) or inhibitory neurotransmitters (GAD, green). B. Imaris Surfaces segmentation produces a rendering of the PV cell through its middle. This tool was also used to render the WFA-labeled PNN surrounding the cell in this example. C. Imaris Spots segmentation allows quantification of inputs from other cells containing GAD (green arrows) or VGluT (magenta arrows) to be visualized and quantified. 